\title{
Using Google Community Mobility Reports to investigate the incidence of COVID-19 in the United States
}

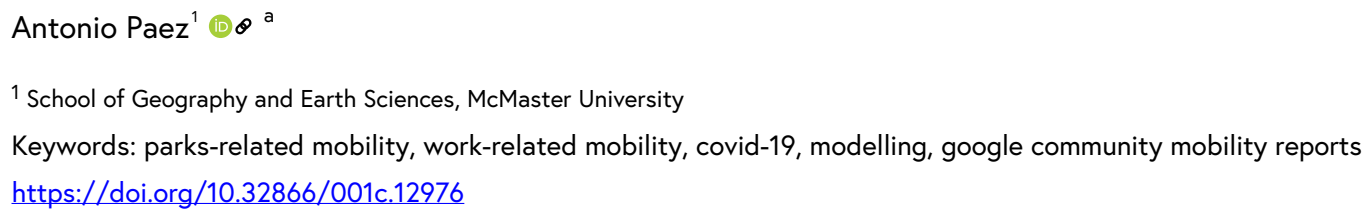

Transport Findings

In 2020 Google released a set of Community Mobility Reports (GCMR). These reports are based on the company's location-tracking capabilities and measure changes in mobility with respect to a baseline. This novel source of data offers an opportunity to investigate potential correlations between mobility and incidence of COVID-19. Using data from the New York Times on COVID-19 cases and GCMR, this paper presents an analysis of mobility levels and incidence of COVID-19 by state in the US. The results provide insights about the utility and interpretability of GCMR for COVID-19 research and decision-making.

\section{RESEARCH QUESTIONS AND HYPOTHESES}

The main policy tool to control the spread of the COVID-19 pandemic has been restrictions to non-essential travel in the form of stay-at-home orders. In the United States, such orders have been implemented on a state-by-state basis with considerable variations in compliance. Concurrently, numerous initiatives have been developed to track the progress and the impact of the pandemic. As a result, there are new sources of data such as the recentlyreleased Google Community Mobility Reports (GCMR) ${ }^{1}$, as well as The New York Times repository of COVID-19 data ${ }^{2}$. These two open data sets offer novel opportunities to investigate in quasi-real time the relationship between mobility patterns and transmission of COVID-19.

This paper investigates the potential of Google Community Mobility Reports to asses the impact of mobility on the incidence of COVID-19. The following questions are posed:

- Do changes in mobility according to GCMR correlate with the incidence of COVID-19?

- And if so, what do we learn about mobility and the spread of the disease?

This paper is a reproducible research document. The source is an $\mathrm{R}$ markdown file available in a public repository ${ }^{3}$.

\footnotetext{
a Corresponding Author Email address: paezha@mcmaster.ca (Antonio Paez)

1 https://www.google.com/covid19/mobility/

2 https://github.com/nytimes/covid-19-data
} 


\begin{tabular}{|c|c|c|c|c|c|}
\hline Variable & Definition & $\min$ & median & $\max$ & sd \\
\hline Incidence & $\begin{array}{l}\text { Total cases of COVID-19 divided by } \\
\text { population (in 100,000s) }\end{array}$ & 0.01 & 56.87 & 1710.06 & 229.76 \\
\hline date & Date & $2020-02-27$ & 2020-04-08 & 2020-05-09 & \\
\hline retail & $\begin{array}{l}\text { Mobility trends for places like restaurants, } \\
\text { cafes, shopping centers, theme parks, } \\
\text { museums, libraries, and movie theaters }\end{array}$ & 0.34 & 0.66 & 1.16 & 0.2 \\
\hline groceries & $\begin{array}{l}\text { Mobility trends for places like grocery } \\
\text { markets, food warehouses, farmers markets, } \\
\text { specialty food shops, drug stores, and } \\
\text { pharmacies }\end{array}$ & 0.66 & 0.93 & 1.26 & 0.12 \\
\hline parks & $\begin{array}{l}\text { Mobility trends for places like local parks, } \\
\text { national parks, public beaches, marinas, dog } \\
\text { parks, plazas, and public gardens }\end{array}$ & 0.36 & 1.15 & 2.28 & 0.29 \\
\hline transit & $\begin{array}{l}\text { Mobility trends for places like public transport } \\
\text { hubs such as subway, bus, and train stations }\end{array}$ & 0.24 & 0.7 & 1.14 & 0.22 \\
\hline work & Mobility trends for places of work & 0.34 & 0.62 & 1.05 & 0.18 \\
\hline residential & Mobility trends for places of residence & 0.98 & 1.14 & 1.27 & 0.07 \\
\hline
\end{tabular}

\section{METHODS AND DATA}

GCMR use aggregated and anonymized data to chart changes in mobility with respect to different classes of places (see Table 1). Mobility indicators are calculated based on the frequency and length of visits to places. The reports give percentage change from a baseline level, which corresponds to the median value of mobility of identical days of the week during the period between January 3 and Feb 6, 2020. Covid-19 data is compiled by The New York Times based on reports from state and local health agencies.

For analysis, all mobility indicators are centered so that the value of 1 is the baseline mobility, and a 0.01 deviation corresponds to a $1 \%$ change. The incubation time of the disease is between 2 and 12 days (95\% interval; see Lauer et al. (2020)). Given this, it is to be expected that any changes in mobility will have a lagged effect on the discovery of new cases. For this reason, lagged moving averages of the mobility indicators are calculated. Furthermore, it is possible that mobility and reports of new cases of COVID-19 are endogenous, if the public adjust their mobility according to reports of the incidence. Therefore, in addition to being consistent with an incubation period, use of lagged indicators also helps to break this potential endogeneity.

The lagged indicators are calculated as the mean of the mobility indicator using the values from date-minus-12-days to date-minus-2-days. Furthermore, using the cumulative number of reported COVID-19 cases, the incidence is calculated after dividing by the population of the state (in 100,000s). This 
Table 2: Simple correlation between $\log ($ incidence) and the mobility indicators

\begin{tabular}{lccccccc}
\hline & log_incidence & retail & groceries & parks & transit & work & residential \\
\hline log_incidence & 1.00 & -0.86 & -0.73 & -0.20 & -0.82 & -0.91 & 0.89 \\
retail & -0.86 & 1.00 & 0.88 & 0.38 & 0.91 & 0.96 & -0.98 \\
groceries & -0.73 & 0.88 & 1.00 & 0.41 & 0.88 & 0.87 & -0.88 \\
parks & -0.20 & 0.38 & 0.41 & 1.00 & 0.46 & 0.33 & -0.36 \\
transit & -0.82 & 0.91 & 0.88 & 0.46 & 1.00 & 0.92 & -0.93 \\
work & -0.91 & 0.96 & 0.87 & 0.33 & 0.92 & 1.00 & -0.98 \\
residential & 0.89 & -0.98 & -0.88 & -0.36 & -0.93 & -0.98 & 1.00 \\
\hline
\end{tabular}

Note:

All mobility indicators are lagged 11-day moving averages

variable (log-transformed) is paired with the corresponding lagged moving average of the mobility indicators. The log-transformation is useful to avoid negative values of incidence when making predictions. Table 1 shows the descriptive statistics of the data set. Analysis is based on correlation analysis, multivariate regression, and data visualization.

\section{FINDINGS}

Table 2 shows that the mobility indicators are highly correlated with each other. Two variables are selected for multivariate analysis: parks- and workrelated mobility. Work has a high correlation with the outcome variable, and its correlation with parks is relatively weak, which increases the information content of the two variables in multivariate analysis. Furthermore, parks- and work-related mobility represent two dimensions of out-of-home activities: mandatory and discretionary travel.

A regression model is estimated with the log of incidence as the dependent variable. The covariates enter the regression in the form of a second order polynomial expansion. In addition, the date (centered on April 5) is introduced to account for the temporal trend of the pandemic. Finally, an indicator variable for the state of New York is used to distinguish the unusually high incidence of the disease there. The results of the model are shown in Table 3. The model provides a good fit to the data and all variables reported are significant at $p<0.10$ or better.

There is an overall temporal trend that indicates a growing incidence over time, but at a decelerating rate (see negative sign of date ${ }^{\wedge} 2$ ). Mobility related to parks and to work are both associated with higher incidence of COVID-19, however, the effect of parks-related mobility grows non-linearly (see positive sign of quadratic term), whereas the effect of work-related mobility grows at a decreasing rate (see negative sign of quadratic term). Furthermore, the negative sign for the interaction of these two mobility indicators captures the tradeoffs between these two forms of mobility and their impact on incidence. The influence of parks-related mobility was relatively weak early in the pandemic 
Table 3: Results of estimating regression model. Dependent variable is log(Incidence).

\begin{tabular}{lcc}
\hline Variable & Coefficient Estimate & p-value \\
\hline date & 0.1618 & $<0.001$ \\
date $^{\wedge}$ & -0.0008 & 0.0093 \\
parks 2 & 0.2518 & 0.0562 \\
parks & 4.1307 & $<0.001$ \\
parks $x$ work & -8.1480 & $<0.001$ \\
work & 9.4568 & $<0.001$ \\
work^2 & -3.4421 & $<0.001$ \\
parks $x$ date & -0.0948 & $<0.001$ \\
parks $x$ date^2 & 0.0036 & $<0.001$ \\
work $x$ date^2 & -0.0058 & $<0.001$ \\
NY & 1.8627 & $<0.001$ \\
\hline Note: & & \\
Coefficient of Determination $R^{2}=0.972$ & & \\
Adjusted Coefficient of Determination $R^{2}=0.972$ & & \\
Standard Error $\sigma=0.689$ & & \\
\hline
\end{tabular}

(negative sign of the parks $\mathrm{x}$ date term) but has become more important over time (positive sign of the parks $\mathrm{x}$ date^ 2 term). The opposite happens with work-related mobility, the importance of which has declined over time (negative sign of work $\mathrm{x}$ date^2 term). As seen in the table, incidence of COVID-19 in New York is consistently higher.

Visualization is the most effective way to understand the trend according to the mobility indicators and date. Figure 1 shows the prediction surfaces on four different dates at intervals of 15 days: March 21, when the first states began implementing stay-at-home orders; then April 5, two weeks into the lockdown; this is followed by April 20, at a time when some states started to consider relaxing stay-at-home orders; and finally May 5, when some states were reopening and/or letting stay-at-home orders lapse.

On March 21 there were still only minor departures from the baseline level of mobility (recall that these are temporally lagged); the prediction surface at this point is relatively flat. This changes by April 5, when work-based mobility has declined substantially. Although every state registers lower work-based mobility, there are large variations in parks-based mobility, with some states seeing increases of up to $60 \%$ for this class of mobility. By May 5, park-related mobility in some states had increased to $100 \%$ of the baseline.

The prediction surfaces are hyperbolic paraboloids on any given date, and in general indicate an expectation of higher incidences as either class of mobility increases, but with a progressively steeper trend for park-based mobility over time. On the last date examined, May 5, the trend becomes more steep for park-based mobility, even as this indicator continues to display large variations 
March 21, 2020
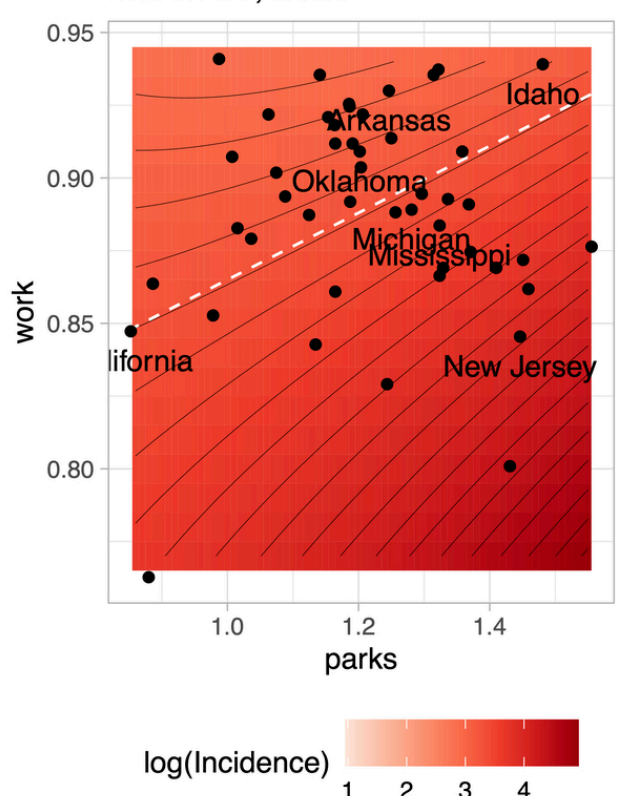

April 20, 2020

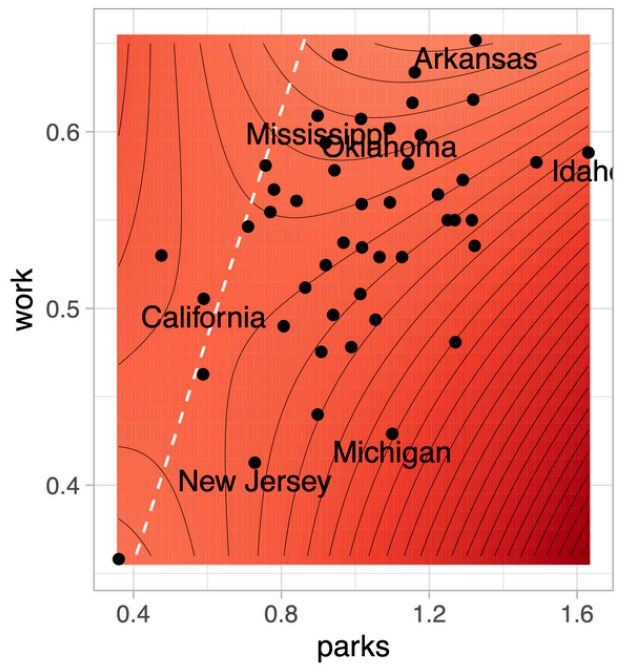

$\log ($ Incidence $)$
April 05, 2020

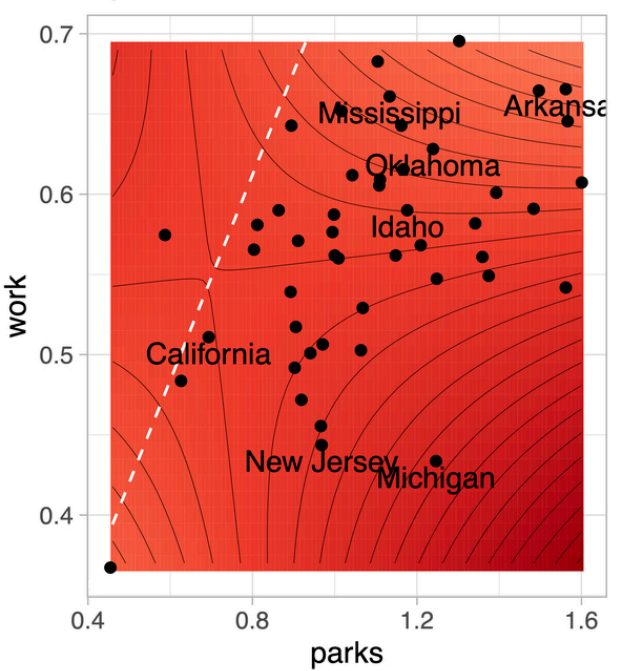

$\log ($ Incidence)

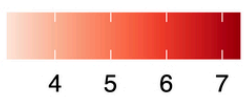

May 05, 2020

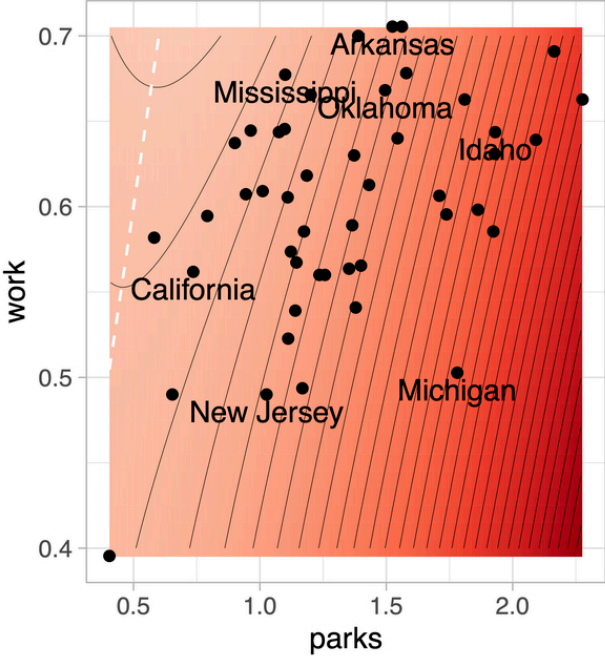

$\log ($ Incidence $)$

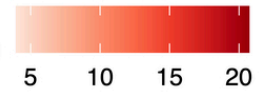

Figure 1

Prediction surfaces at three points during the pandemic according to the model; the dots are a scatterplot of the parks- and work-related mobility indicators of the states on that date; the white dashed line is the fold of the saddle.

from the baseline in both directions. The white dashed lines in the plots are the folds of the saddles, and represent, for each date, the combination of parks- and work-related mobility levels that tended to minimize the incidence.

The results indicate that over time the benefits of reduced work-related mobility can be easily offset by parks-related mobility. For example, California has consistently registered lower levels of parks-related mobility whereas Idaho has had high levels of this kind of mobility throughout the pandemic. The 
incidence of COVID-19 grew in the intervening period; however, between March 21 and May 5 growth in incidence in California was $876.42 \%$ whereas Idaho's growth in incidence over the same period was $2519.05 \%$.

These results suggest the potential of GCMR to investigate the potential effects of mobility on the incidence of COVID-19. In particular, growth appears to be more strongly driven by parks-related mobility. In terms of the use of these mobility indicators, there are some limitations that must be acknowledged. The baseline level is not defined in a metric that is amenable to policy development (e.g., person-km travelled). Without a clearer understanding of the absolute levels of these variables, these indicators are useful for inference and perhaps short-term forecasting, but their potential for applied policy analysis appears to be more limited. 


\section{REFERENCES}

Lauer, Stephen A., Kyra H. Grantz, Qifang Bi, Forrest K. Jones, Qulu Zheng, Hannah R.

Meredith, Andrew S. Azman, Nicholas G. Reich, and Justin Lessler. 2020. "The Incubation Period of Coronavirus Disease 2019 (Covid-19) from Publicly Reported Confirmed Cases: Estimation and Application." Annals of Internal Medicine 172 (9): 577-82. https://doi.org/10.7326/ $\underline{\mathrm{m} 20-0504}$. 


\section{FIGURES, TABLES, AND SUPPLEMENTARY MATERIALS}

1117003_using_google_community_mobility_reports_to_investigate_the_incidence_of_covid_19 Download: https://transportfindings.org/article/12976-using-google-community-mobility-reports-toinvestigate-the-incidence-of-covid-19-in-the-united-states/attachment/35019.pdf 